\title{
Thoracoscope and thoracotomy in the treatment of thoracic trauma
}

\author{
Juan Shi ${ }^{1}$, Yucun Wang ${ }^{2}$, Wenzhen Geng ${ }^{3}$
}

\begin{abstract}
Objective: To compare clinical effects of thoracoscopic surgery and thoracotomy in the treatment of thoracic trauma.

Methods: Two hundred and fourteen patients with thoracic trauma were randomly divided into a control group and an observation group, 107 in each group. The control group was treated with conventional thoracotomy, while the observation group was treated with thoracoscopic surgery. The operation-related indications, hospitalization, postoperative complications and inflammatory factor level were observed and compared between the two groups. The study was conducted from April 2016 to February 2018.

Results: The duration of operation of the observation group was shorter than that of the control group, the amount of bleeding during operation of the observation group was less than that of the control group, and the postoperative visual analogue score (VAS) of the observation group was lower than that of the control group; the difference were statistically significant $(P<0.05)$. The hospitalization time, time of off-bed activity and time of resuming daily life of the observation group were shorter than those of the control group, and the amount of drainage fluid of the observation group within 24 hours after operation was less than that of the control group; the differences had statistical significance $(P<0.05)$. There was no significant difference in the incidence of postoperative complications between the two groups $(P>0.05)$. The levels of C-reactive protein (CRP), tumor necrosis factor (TNF)-a and interleukin (IL)- 6 in both groups after surgery were higher than those before surgery, but the indicators in the observation group were lower than those in the control group $(\mathrm{P}<0.05)$.

Conclusion: Thoracoscopic surgery can reduce pains of patients, speed up recovery, and reduce incidence of surgical infection in the treatment of thoracic trauma. It is a safe and effective treatment method, which is worth clinical application.
\end{abstract}

KEYWORDS: Thoracic trauma, Thoracoscopic surgery, Thoracotomy.

doi: https://doi.org/10.12669/pjms.35.5.514

How to cite this:

Shi J, Wang Y, Geng W. Thoracoscope and thoracotomy in the treatment of thoracic trauma. Pak J Med Sci. 2019;35(5):1238-1242. doi: https://doi.org/10.12669/pjms.35.5.514

This is an Open Access article distributed under the terms of the Creative Commons Attribution License (http://creativecommons.org/licenses/by/3.0), which permits unrestricted use, distribution, and reproduction in any medium, provided the original work is properly cited.

1. Juan Shi,

Department of Cardiothoracic Surgery,

2. Yucun Wang,

Department of Rheumatology and Immunology,

3. Wenzhen Geng,

Department of Cardiovascular Medicine,

1-3: Binzhou People's Hospital,

Shandong, 256610, China.

Correspondence:

Juan Shi,

No.515 Huanghe $7^{\text {th }}$ Road,

Binzhou People's Hospital, Shandong 256610, China.

Email: jshijuan@yeah.net

* Received for Publication:

* Revision Received:

* Revision Accepted:

February 5, 2019

July 11,2019

July 13, 2019

\section{INTRODUCTION}

Thoracic trauma which is common in clinic is mainly caused by direct violence such as instrument injury, collision injury, falling injury and so on, which may cause different degrees of thoracic trauma. Sites of thoracic trauma are diverse and complex. Severe patients may even suffer from multiple organ injury, having risks of shock and death. ${ }^{1,2}$ Once thoracic trauma appears, pulmonary contusion and laceration and intrathoracic hemorrhage may be induced, and most patients may also have symptoms such as fracture, contusion, pulmonary infection, chest deformity 
and atelectasis, which can aggravate injury., $\mathrm{A}$ survey has shown that trauma is the leading cause of death among people under 45 years worldwide and chest trauma accounts for $1 / 4 .^{5}$ In the past, thoracic trauma was usually treated by surgical methods. ${ }^{6}$ However, the traditional thoracotomy is limited in application as it can lead to physical and mental trauma and induce many postoperative complications. ${ }^{7,8}$

With the continuous development of thoracoscopic surgery in recent years, its application in the detection and treatment of thoracic injury has become increasingly widespread. ${ }^{9}$ A study has shown that thoracoscopic surgery is superior to traditional thoracotomy in reducing postoperative complications and shortening hospital stay besides advantages of small incision and less pain. ${ }^{10}$ However, the sample size of current studies concerning thoracoscopic treatment for thoracic trauma is small. In addition, thoracoscopy will increase incidence of complications such as pneumonia, atelectasis or iatrogenic diaphragmatic hernia. ${ }^{11}$ Therefore, the effect of thoracoscopy on the treatment of thoracic trauma still needs to be explored. In order to further explore the application value of thoracoscopic surgery, clinical effects of thoracoscopic surgery and thoracotomy in the treatment of thoracic trauma were compared.

\section{METHODS}

Two hundred and fourteen patients with thoracic trauma who were admitted to our hospital from April 2016 and February 2018 were selected. The trauma of the patients was scored using abbreviated injury scale (AIS) and injury severity score (ISS). AIS score no lower than three points or ISS no lower than 16 points was evaluated as severe thoracic trauma. Patients with AIS score no lower than three points and ISS no lower than 16 points, needed surgical treatment, had stable haemodynamics were included. Those who had mild disease condition that could be treated by non-surgical treatment and patients who themselves or whose family members did not wish to participate in the study were excluded. There were 127 males and 87 females, and they aged 19 72 years (average $51.3 \pm 8.0$ years). They were numbered using random number table and divided into two groups, 107 each. The general data of the two groups had no significant difference, as shown in Table-I. The study was carried out after being approved by the ethics committee of our hospital.
Patients in the control group received traditional thoracotomy. After total intravenous anesthesia, proper surgical incision was made according to the position of injury. Relevant treatment was made after entering into the thoracic cavity, and the specific treatment way was consistent with the observation group. Drainage tube was inserted after surgery, and the incision was sutured. Patients in the observation group received thoracoscopic surgery. Firstly, general anesthesia was performed, and patients were given double-lumen tube (specification: Fr28; Shengguang Medical Products Co., Ltd., China) intubation. The patents kept a lateral position. The $7^{\text {th }}$ intercostal space along the midaxillary line was taken as the surgical approach. The general condition inside the thoracic cavity was probed using the thoracoscope. Blood clot inside the thoracic cavity was cleaned. The injury of the lung was examined. The lung was sutured through incision at the fourth intercostal space under the armpit. Resection was made if the lacerated wound is too severe that could not be repaired. Whether there was other organ injury or bleeding was checked. After repair and hemostasis, the site with rib fracture was fixed under the thoracoscope. The site and number of rib fracture were observed carefully. The rib was pressed under the thoracoscope to find the site of sternal incision suitable for auxiliary fixation. The thoracic walls were cut open layer by layer. The broken ends of fractured bone were exposed under an incision as small as possible. The periosteum of the broken ends was stripped for $2 \sim 3 \mathrm{~cm}$, and then anatomical reduction was performed. Damages to the pleura were avoided in the process of operation. Bone lamella was encircled using proper memory ring and covered both ends of the rib after disinfection. The thoracic cavity was washed if the fixation was satisfactory and there was no bleeding. One drainage tube was left.

After surgery, patients in the two groups were sent to intensive care unit and given conventional nursing cares. The changes of vital signs were closely monitored, including pulse, heart rate, consciousness and pupil. Anti-infection, fluid infusion and oxygen nursing were positively given. The treatment and nursing of patients strictly followed aseptic operation principles. Medicine was changed every day. Firstly, the injury site was disinfected and then covered with local transparent adhesive. Patients with local bleeding were in time, and thrombin was used for reducing post-operative traumatic infection. The respiratory function of patients was observed. Blood gas analysis was monitored. Ventilator parameters 
Table-I: Baseline data between the two groups.

\begin{tabular}{|c|c|c|c|c|c|}
\hline \multicolumn{2}{|l|}{ Groups } & Dservation group & Control group & $t / X^{2}$ & $P$ \\
\hline \multicolumn{2}{|l|}{ Sex ratio (male/female) } & $66 / 41$ & $61 / 46$ & 1.083 & $>0.05$ \\
\hline \multicolumn{2}{|l|}{ Age (year) } & $52.15 \pm 8.4$ & $50.37 \pm 8.1$ & 0.397 & $>0.05$ \\
\hline \multicolumn{2}{|l|}{ ISS } & $23.66 \pm 2.32$ & $24.16 \pm 2.08$ & 0.264 & $>0.05$ \\
\hline \multicolumn{2}{|c|}{ Time between onset and diagnosis $(\mathrm{h})$} & $6.72 \pm 2.15$ & $6.81 \pm 2.34$ & 0.892 & $>0.05$ \\
\hline \multirow[t]{4}{*}{ Causes for injury $[\mathrm{n}(\%)]$} & Traffic & 56 & 54 & 0.843 & $>0.05$ \\
\hline & Fighting & 27 & 28 & & \\
\hline & Fall & 21 & 20 & & \\
\hline & Accidents at construction site & 3 & 5 & & \\
\hline
\end{tabular}

were adjusted to enhance respiratory tract care to prevent respiratory tract infection. The patients were turned over regularly, and expectoration was paid attention to. After extubation, oxygen inhalation mask was used to ensure that patients breathe smoothly. Nursing of complications was done. Abnormal condition was reported to doctors for timely and effective treatment.

Observational indicators: Duration of operation and intraoperative bleeding amount were recorded. The postoperative pain of patients was evaluated using visual analogue scale. The hospitalization time, time of off-bed activity, time of daily living recovery, postoperative 24-hour drainage volume and occurrence of complications were recorded. Levels of C-reactive protein (CRP), tumor necrosis factor (TNF)- $\alpha$ and interleukin (IL)- 6 were compared before surgery and 24 hour after surgery.

Statistical Analysis: Data were statistically analyzed using SPSS ver. 20.0. Measurement data were processed by normality test and expressed as Mean $\pm S D$ if satisfying normal distribution. Data were compared between the two groups using independent sample t-test. Data within the same group before and after treatment was compared using paired t-test. Data which did not satisfy normal distribution was processed by rank sum test. Enumeration data were expressed by frequency; comparison between groups was performed using Chi-square test. $\mathrm{P}<0.05$ indicates that difference had statistical significance.

\section{RESULTS}

The duration of operation of the observation group was shorter than that of the control group, and the intraoperative bleeding amount of the observation group was less than that of the control group, and the postoperative VAS score of the observation group was lower than that of the control group. Differences were statistically significant $(\mathrm{P}<0.05$, Table-II).

The hospitalization time, time of off-bed activity and time of daily living recovery of the observation group were shorter than those of the control group. The postoperative 24-hour drainage volume of the observation group was less than that of the control group. There were significant differences $(\mathrm{P}<0.05$, Table-III).

Levels of IL-6, TNF-a and CRP of both group after operation were higher than those before operation, but the levels of the observation group were significantly lower than those of the control group. Differences had statistical significance $(\mathrm{P}<0.05$, Table-IV).

Table-II: Surgical indicators between the two groups.

\begin{tabular}{lcccc}
\hline Groups & Observation group & Control group & $t$ & $P$ \\
\hline Duration of operation $(\mathrm{min})$ & $45.91 \pm 23.62$ & $95.63 \pm 34.07$ & 13.857 & $<0.05$ \\
Intraoperative bleeding amount $(\mathrm{mL})$ & $172.94 \pm 134.51$ & $351.42 \pm 78.79$ & 19.476 & $<0.05$ \\
VAS score (point) & $3.52 \pm 1.13$ & $7.19 \pm 8.16$ & 6.931 & $<0.05$ \\
\hline
\end{tabular}

Table-III: Hospitalization indicators between the two groups.

\begin{tabular}{lcccc}
\hline Groups & Observation group & Control group & $t$ & $P$ \\
\hline Hospitalization time $(\mathrm{d})$ & $6.14 \pm 2.08$ & $8.15 \pm 1.26$ & 8.258 & $<0.05$ \\
Time of off-bed activity $(\mathrm{d})$ & $1.68 \pm 0.24$ & $3.26 \pm 1.06$ & 9.531 & $<0.05$ \\
Time of daily living recovery $(\mathrm{d})$ & $9.57 \pm 1.26$ & $17.27 \pm 3.63$ & 17.37 & $<0.05$ \\
Postoperative 24-h drainage volume $(\mathrm{mL})$ & $253.22 \pm 52.13$ & $280.33 \pm 52.30$ & 15.216 & $<0.05$ \\
\hline
\end{tabular}

Pak J Med Sci September - October 2019 Vol. 35 No. 5 www.pjms.org.pk 1240 
Juan Shi et al.

Table-IV: Levels of inflammatory factors between the two groups before and after operation.

\begin{tabular}{lcccc}
\hline \multirow{2}{*}{ Groups } & \multicolumn{2}{c}{ Observation group } & \multicolumn{2}{c}{ Control group } \\
\cline { 2 - 5 } & Before operation & After operation & Before operation & After operation \\
\hline IL-6 (UG/L) & $38.21 \pm 8.31$ & $54.24 \pm 12.35^{* \#}$ & $39.11 \pm 7.42$ & $82.54 \pm 7.41^{*}$ \\
TNF-a (UG/L) & $17.45 \pm 5.31$ & $65.30 \pm 14.53^{* \#}$ & $16.65 \pm 3.36$ & $87.56 \pm 21.36^{*}$ \\
CRP $(\mathrm{mg} / \mathrm{L})$ & $7.58 \pm 1.20$ & $45.36 \pm 11.23^{*}$ & $7.65 \pm 1.42$ & $67.20 \pm 11.27^{*}$ \\
\hline
\end{tabular}

Note: *suggests $\mathrm{P}<0.05$ comparing to before operation, \#suggests $\mathrm{P}<0.05$ comparing to the control group.

One patient had acute respiratory distress syndrome and one patient had pulmonary infection in the observation group; the incidence of complications was $1.87 \%$. One patient had acute respiratory distress syndrome, one patient had shock and one patient had pulmonary infection in the control group; the incidence of complications was $2.80 \%$. The difference of incidence of complications between the two groups was not statistically significant $(\mathrm{P}>0.05)$. All the complications disappeared after symptomatic treatment.

\section{DISCUSSION}

In recent years, the incidence of chest traumatic diseases is increasing year by year. There are many factors leading to chest trauma, including industrial and mining, transportation, natural disasters and so on. ${ }^{12}$ Moreover, chest trauma will seriously affect their respiratory and circulatory functions, lead to a series of pathological changes, and even threaten lives in severe cases. ${ }^{13}$ Surgery is the preferred method for patients with thoracic trauma, most of which are treated by traditional thoracotomy. However, thoracotomy has many disadvantages, such as large trauma, multiple complications and slow recovery, which will not only cause great trauma to the body, but also aggravate pains. ${ }^{14}$ Traditional treatments also include local bandage and assisted thoracic drainage, which can stabilize the injured chest wall and drain the fluid from the chest. However, limited by pains and respiratory movement, patients cannot cough up sputum in the body and may suffer from pulmonary infection and other complications, which may aggravate the disease.${ }^{15}$ Therefore, it is necessary to find an ideal way to treat chest trauma.

With the continuous development of medical technology, a variety of minimally invasive technologies has developed and has been widely used in clinical practice. Video-assisted thoracoscopy has been recognized by many scholars for its advantages of small trauma and rapid recovery. Thoracoscopic surgery can insert thoracoscope through a new incision or the original closed thoracic drainage opening. ${ }^{16}$ Thoracoscopy can help more intuitively and clearly understand organs such as diaphragm, mediastinum, chest wall and heart and observe whether there is any injury and the actual severity of injury and operation can be implemented according to thoracoscopic results. In terms of operative field, thoracoscopic surgery is better than thoracotomy. ${ }^{17}$ In the traditional thoracotomy, it is usually necessary to make a general judgment on the location of thoracic injury and then determine the surgical incision before surgery. For thoracoscopic surgery, the position of the entrance for thoracoscope is usually fixed, and the condition of thoracic injury will not affect the intraoperative exploration and operation. In addition, incision can be made according to the actual probing result of thoracoscope, which can effectively simplify the operation process and make the whole operation more convenient and fast. ${ }^{18}$ In a study ${ }_{19}^{19} 24$ patients with posttraumatic hemothorax were treated by thoracoscopic surgery. It was found that thoracoscopic surgery could significantly reduce injuries caused by treatment and the physiological interference caused by operation and it led to a low recurrence rate and few complications. The results of this study also found that patients who received thoracoscopic surgery had shorter operation time and hospitalization time, less intraoperative bleeding amount, lower VAS score and better recovery after operation compared to those who received traditional thoracotomy, which was similar to the results of $\mathrm{Xu} \mathrm{L}$ et al. ${ }^{20}$ It shows that thoracoscopic surgery can reduce operation time and intraoperative bleeding volume and has a significant promotion effect on the improvement of body functions.

Basic medicine points out that the inflammatory state of body will directly affect operation. If levels of inflammatory factors win the body were too high, it will increase risks of infection and even develop into systemic inflammatory response syndrome which can cause multiple organ dysfunction and is also an important cause of death. ${ }^{21}$ Therefore, 
in surgical treatment, the over-activation of inflammatory cells should be paid attention to, surgical trauma should be reduced as far as possible, and complications should be reduced. The results of this study showed that levels of IL-6, TNF-a and CRP in the two groups were higher than those before operation, but the levels of these indicators in the observation group were significantly lower than those in the control group. The results were similar to Raghabendran $\mathrm{K}$ et al. ${ }^{22}$ It shows that patients who receive thoracoscopic surgery suffered from smaller stimulation, less loss of immune function and a lower stress level, which is beneficial to postoperative rehabilitation.

Although thoracoscopic technique has many advantages, it also requires doctors to have high operational ability and level. Thoracic cavity should be carefully explored during operation, especially mediastinum, posterior sternum and pericardium which are easy to be missed. It is necessary to carefully explore and study the damage of those parts. For some patients with sternum and thoracic vertebra injuries, thorough hemostasis should be carried out in time. For those patients with aortic injuries, CT imaging should be combined, and thoracotomy can be performed if necessary. Alveolar resection should be performed concurrently for patients with bullae.

\section{CONCLUSION}

Thoracoscopic surgery can reduce surgical injuries to patients, reduce the amount of bleeding and the excessive activation of inflammatory cells, and speed up recovery in the treatment of chest trauma. It is a safe and effective method, which is worth clinical application.

Declaration of interest: All authors declared there was no conflict interests involved.

\section{Grant Support \& Financial Disclosures: None.}

\section{REFERENCES}

1. McNamara C, Mironova I, Lehman E, Olympia RP. Predictors of intrathoracic injury after blunt torso trauma in children presenting to an emergency department as trauma activations. J Emerg Med. 2017; 52(6):793-800. doi: 10.1016/j.jemermed.2016.11.031.

2. Qi YJ. Clinical study on VATS combined mechanical ventilation treatment of ARDS secondary to severe chest trauma. Exp Ther Med. 2016;12(2):1034-1038. doi: 10.3892/etm.2016.3355.

3. Huang FD, Yeh WB, Chen SS, Liu YY, Lu IY, Chou YP, Wu TC. Early management of retained hemothorax in blunt head and chest trauma. World J Surg. 2018;42(7):2061-2066. doi: 10.1007/s00268017-4420-x.

4. Tarng YW, Liu YY, Huang FD, Lin HL, Wu TC, Chou YP. The surgical stabilization of multiple rib fractures using titanium elastic nail in blunt chest trauma with acute respiratory failure. Surg Endosc. 2016;30(1):388-395. doi: 10.1007/s00464-015-4207-9.
5. Billeter AT, Druen D, Franklin GA, Smith JW, Wrightson W Richardson JD. Video-assisted thoracoscopy as an important tool for trauma surgeons: a systematic review. Langenbeck's Arch Surg. 2013;398(4):515-523. doi: 10.1007/s00423-012-1016-7.

6. Ma HS, Ma JH, Xue FL, Fu XN, Zhang N. Clinical analysis of thoracoscopic surgery combined with intraoperative autologous blood transfusion in the treatment of traumatic hemothorax. Chin J Traumatol. 2016;19(6):371-372.

7. Refaely Y, Koyfman L, Friger M, Ruderman L, Saleh MA, Sahar $\mathrm{G}$, et al. Clinical outcome of urgent thoracotomy in patients with penetrating and blunt chest trauma: a retrospective survey. Thorac Cardiovasc Surg. 2018;66(8):686-692. doi: 10.1055/s-0037-1608899.

8. Morley EJ, Johnson S, Leibner E, Shahid J. Emergency department evaluation and management of blunt chest and lung trauma (Trauma CME). Emerg Med Pract. 2016;18(6):1-20.

9. Chiang TY, Yin MF, Yang SM, Chen KC. Thoracoscopic management of incarcerated lung herniation after blunt chest trauma: a case report and literature review. J Thorac Dis. 2017;9(3):E253. doi: 10.21037/ jtd.2017.03.41.

10. Sesma J, Alvarez M, Lirio F, Galvez C, Galiana M, Baschwitz $\mathrm{B}$, et al. Single-incision video-assisted thoracoscopic evaluation and emergent surgery for severe lung and chest wall injury after thoracic trauma in a water park. Ann Transl Med. 2017;5(16):328. doi: 10.21037/atm.2017.05.12.

11. Wang PG, Xue F. Influence of thoracoscopic operation on clinical indexes of patients with traumatic hemopneumothorax. J Laparosc Surg. 2016;21(9):666-669. doi: 10.13499/j.cnki.fqjwkzz.2016.09.666.

12. Narvestad JK, Meskinfamfard M, Soreide K. Emergency resuscitative thoracotomy performed in European civilian trauma patients with blunt or penetrating injuries: a systematic review. Eur J Trauma Emerg Surg. 2016;42(6):677-685. doi: 10.1007/s00068-015-0559-z.

13. Van Vledder MG, Van Waes OJF, Kooij FO, Peters JH, Van Lieshout EMM, Verhofstad MHJ. Out of hospital thoracotomy for cardiac arrest after penetrating thoracic trauma. Injury. 2017;48(9):1865-1869. doi: 10.1016/j.injury.2017.04.002.

14. Dayama A, Sugano D, Spielman D, Stone ME Jr, Kaban J, Mahmoud A, et al. Basic data underlying clinical decision-making and outcomes in emergency department thoracotomy: tabular review. Anz J Surg. 2016;86(1-2):21-26. doi: 10.1111/ans.13227.

15. Moskowitz EE, Burlew CC, Kulungowski AM, Bensard DD Survival after emergency department thoracotomy in the pediatric trauma population: a review of published data. Pediatr Surg Int. 2018;34(8):857-860. doi: 10.1007/s00383-018-4290-9.

16. EH Fouly, Z Ashraf, M Ghalwash. Thoracoscopy versus thoracotomy in hemodynamically stable patients with closed thoracic trauma. J Egypt Soc Cardio-Thorac Surg. 2018;26(1):64-67.

17. Schots JP, Vissers YL, Hulsewe KW, Meesters B, Hustinx PA, Pijnenburg A, et al. Addition of video-assisted thoracoscopic surgery to the treatment of flail chest. Ann Thorac Surg. 2017;103(3):940-944. doi: 10.1016/j.athoracsur.2016.09.036.

18. Chiang TY, Yin MF, Yang SM, Chen KC. Thoracoscopic management of incarcerated lung herniation after blunt chest trauma: a case report and literature review. J Thorac Dis. 2017;9(3):E253-E257. doi: 10.21037/jtd.2017.03.41

19. Kang PM, Tan QY, Wang RW, Deng B, Zhou JH, Tao SL. Videoassisted thoracic surgery for thoracic trauma in 56 patients. J Traum Surg. 2014;16(4):297-300.

20. Xu L, Chang HL, Chang M. Analysis of clinical effect of videoassisted thoracoscopic surgery in the treatment of traumatic hemopneumothorax. Clin Res Pract. 2016;1(16):97.

21. Chen Y. Application value of thoracoscope in surgical treatment of thoracic trauma. Mod Diag Treat. 2015;26(21):4963-4964.

22. Raghavendran K, Davidson BA, Woytash JA, Helinski JD, Marschke CJ, Manderscheid PA, et al. The evolution of isolated bilateral lung contusion from blunt chest trauma in rats: cellular and cytokine responses. Shock. 2005;24(2):132-138.

Authors' Contribution: JS \& YCW: Study design, data collection and analysis. JS \& WZG: Manuscript preparation, drafting and revising. JS: Review and final approval of manuscript. 